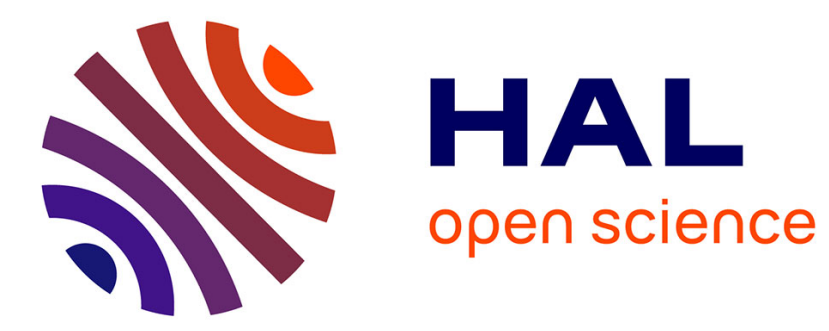

\title{
Amphiphilic polymers based on polyoxazoline as relevant nanovectors for photodynamic therapy
}

Amandine Oudin, Julie Chauvin, Laure Gibot, Marie-Pierre Rols, Stephanie

Balor, Dominique Goudounèche, Bruno Payre, Barbara Lonetti, Patricia

Vicendo, Anne-Françoise Mingotaud, et al.

\section{To cite this version:}

Amandine Oudin, Julie Chauvin, Laure Gibot, Marie-Pierre Rols, Stephanie Balor, et al.. Amphiphilic polymers based on polyoxazoline as relevant nanovectors for photodynamic therapy. Journal of materials chemistry B, 2019, 7 (32), pp.4973-4982. 10.1039/c9tb00118b . hal-02276059

\section{HAL Id: hal-02276059 \\ https://hal.science/hal-02276059}

Submitted on 22 Mar 2021

HAL is a multi-disciplinary open access archive for the deposit and dissemination of scientific research documents, whether they are published or not. The documents may come from teaching and research institutions in France or abroad, or from public or private research centers.
L'archive ouverte pluridisciplinaire HAL, est destinée au dépôt et à la diffusion de documents scientifiques de niveau recherche, publiés ou non, émanant des établissements d'enseignement et de recherche français ou étrangers, des laboratoires publics ou privés. 


\title{
Amphiphilic polymers based on Polyoxazoline as relevant nanovectors for Photodynamic Therapy
}

\author{
Amandine Oudin, ${ }^{a}$ b Julie Chauvina, Laure Gibot a, c, Marie-Pierre Rols ${ }^{c}$, Stéphanie Balor ${ }^{d}$, \\ Dominique Goudounèche ${ }^{e}$, Bruno Payrée, Barbara Lonettia ${ }^{a}$, Patricia Vicendo ${ }^{a}$, Anne-Françoise \\ Mingotaud $^{\mathrm{a},{ }^{*}}$ and Vincent Lapinte ${ }^{\mathrm{b}, *}$

\begin{abstract}
An amphiphilic polymer (CmPOX) based on poly(2-methyl-2-oxazoline) linked to a hydrophobic part composed of an polymeric self-assemblies, typically of ca. $10 \mathrm{~nm}$ in size which were characterized by TEM, cryo-TEM and DLS. The nanocarriers were further formulated to yield photo-crosslinked systems by dimerization of coumarin units of a coumarinfunctionalized poly(methyl methacrylate) (CmPMMA) and CmPOX. The formed vectors were used to encapsulate Pheophorbide a, a known photosensitizer for PhotoDynamic Therapy. Cytotoxicity as well as photooxicity experiments led in vitro on human tumor cells revealed the great potential of these nanovectors for photodynamic therapy.
\end{abstract} \\ aliphatic chain ended by a photo-active coumarin group has been synthesized. It exhibits the ability of forming small
}

\section{Introduction}

In various scientific fields, medicine included, an external control of the desired process is often looked for. From this standpoint, light is an interesting stimulus, since it can be easily switched on/off, be very localized and penetrate between micrometers and centimeters of tissue depending on the wavelength used. With the development of a large variety of medical lasers, photomedicine has therefore strongly progressed in the last 30 years. Among the techniques of photomedicine, photodynamic therapy (PDT) is a clinically approved technique using photosensitizers which yields Reactive Oxygen Species (ROS) upon irradiation, followed by cell death. Since irradiation is a typical local stimulus, PDT provides localized treatment which is highly desirable in many illnesses in dermatology, ophthalmology or oncology. A limiting step however is that the photosensitizer should be specifically distributed in the area to be treated and this is rarely the case after intra-venous injection. Uncontrolled biodistribution leads to an adverse effect, a

a. Laboratoire des IMRCP, Université de Toulouse, CNRS UMR 5623, Université Paul Sabatier, 118 route de Narbonne, F-31062, Toulouse Cedex 9, France.

b. Institut Charles Gerhardt Montpellier UMR5253 CNRS-UM-ENSCM, Equipe Ingénierie et Architectures Macromoléculaires, Université Montpellier - Bat. 17cc1702, Place Eugène Bataillon, F-34095 Montpellier cedex 5, France

c. Institut de Pharmacologie et Biologie Structurale, IPBS, Université de Toulouse, CNRS, UPS, Toulouse, France

d. Microscopie Electronique Intégrative, Centre de Biologie Intégrative, 118 Rte de Narbonne, 31062 Toulouse, France

e. Centre de Microscopie Electronique Appliquée à la Biologie, Faculté de Médecine Toulouse Rangueil, Université de Toulouse, 133, route de Narbonne, 31062 Toulouse cedex 4, France

+ Footnotes relating to the title and/or authors should appear here.

Electronic Supplementary Information (ESI) available: Experimental details and characterizations. cutaneous photosensitivity of the patient during several days. Because such uncontrolled biodistribution of drugs is a central drawback of many treatments and not only for PDT, the last twenty years have seen a burst of studies aiming at developing nanovectors to improve the therapeutic efficiency of known drugs ${ }^{1-3}$. This was partly legitimized by the discovery of so called "Enhanced Permeability and Retention" "(EPR) effect by Maeda in the late eighties 4, 5. This phenomenon showed that, owing to the presence of disjunctions between endothelial cells in vessels close to solid tumors and the low efficiency of lymphatic drainage, some nanovectors could be used to carry drugs in a passive targeting manner. A required specification for this passive targeting is that the vectors should travel undetected in the blood stream as long as they did not reach the tumor site. Among all nanocarrier surface compositions assessed, poly(ethylene oxide) (PEO) appeared to be the most efficient and is currently the polymer most widely used for nanovectors.

In this context, PDT has also been evaluated through the tools of nanomedicine and more especially polymeric nanovectors ${ }^{6}$. Various systems have thus been examined, based on polyesters, polyacrylates, polyacrylamides or peptides... Our team has formerly described the encapsulation of a photosensitizer, namely Pheophorbide $a$, in various polymeric micelles and polymersomes ${ }^{7}, 8$, showing in vitro an improvement of photosensitizer delivery and antitumoral activity after light irradiation. Interestingly, crosslinked vectors were even more efficient ${ }^{9}$. Some of the most recent studies in the literature examined high-performance vectors, compensating the relative hypoxia of tumors ${ }^{10-12}$. Among all the existing literature on polymeric nanovectors used for PDT, ca. $90 \%$ is based on PEO hydrophilic blocks, very much like all other nanomedicine applications of nanovectors. Only very scarcely is another type used, such as polysaccharide ${ }^{13-15}$. 
Ten years ago, some studies began to show that PEO might not be the suggested "gold standard"16, as revealed by the occurrence of accelerated blood clearance $(A B C)$ upon several injections, possible immune responses leading to the loss of the EPR effect after a second injection. Over the last ten years, many studies on this point have been published and it is still the subject of a debate ${ }^{17-19}$. This has led to further evaluation of other polymer types, and a promising lead is poly(2-alkyl-2-oxazoline)s (POx) ${ }^{20}$. Indeed, this family of polymers offers the advantage of exhibiting different hydrophilicities depending on the alkyl group. An excellent recent review by Luxenhofer on poly(2-alkyl-2-oxazoline)s in biomaterials summarized some data on their toxicity, degradability, stealth properties, immunogenicity and biodistribution ${ }^{21}$. Different POx structures have been examined, such as partially hydrolyzed POx, random and block copolymers of cationic and non-ionic POX ${ }^{22}$. Regarding accelerated blood clearance and immunogenicity of poly(2-alkyl-2-oxazolines), conflicting results have been published. Szoka showed an ABC for PEG and POx liposomes ${ }^{23}$, whereas Wyffels and Hoogenboom found another POx-based system to be non-immunogenic ${ }^{24}$. Owing to the need of controlling the state of the nanovectors, photostimulation has been often estimated, and this is also true for POx systems, with different examples of grafted chromophores and the most often aim being photocrosslinking for tissue engineering ${ }^{25-27}$. Interestingly, more than 20 years ago, Saegusa followed by Chujo described the deamidation of poly(2-methyl-oxazoline) followed by grafting of carboxylated-coumarin groups for the formation of gels ${ }^{28,29}$. Coumarin is a benzopyrone which can be reversibly dimerized upon irradiation.

These biomedical examples using POx platform demonstrate the possible strength of this class of polymers and its versatility. It is however surprising that among all these studies, only one example so far has assessed POx polymers as a possible platform for PDT nanovectors. In 2010, Lai described the synthesis of poly(2-ethyl-2-oxazoline-b-D,L-lactide) to encapsulate the photosensitizer metatetra(hydroxyphenyl)chlorin ${ }^{30}$. They did not observe any PDT improvement by encapsulation, emphasized the possible $\mathrm{pH}$ dependent release of the photosensitizer and showed in vivo that cutaneous photosensitivity was decreased by the encapsulation.

Based on this lack of examples in the literature, the assessment of POx as a possible photosensitizer nanovector was relevant. We therefore present here poly(2-methyl-2oxazoline) self-assemblies as potential Pheophorbide a carrier. Since this polymer is hydrophilic, self-assemblies were generated after chemical modification, introducing an alkyl chain, as reported in a previous example ${ }^{31}$. To further examine the effect of crosslinking on this type of vector, a coumarin photoreactive group was also introduced. Beside its possible photodimerization, it is also known to exhibit anti-cancer activities and many derivatives have been reached for this ${ }^{32}$, 33. The use of such a functional moiety in the formulation of a nanovector could thus bring a synergistic effect to PDT.

Furthermore, another originality of our work lies in the use of a coumarin-bearing oligomethacrylate chain to guarantee the photocrosslinking of the entire self-assembly. This concept could also be extended and the oligomethacrylate chain used to easily include in the crosslinked nanovector other functionalities.

\section{Materials and methods}

4-Methylumbelliferone, 11-bromoundecanol, diethylether, ethanol, acetone, $\mathrm{p}$-toluenesulfonyle chloride, piperidine, pyridine, $\mathrm{MgSO}_{4}$, potassium carbonate $\left(\mathrm{K}_{2} \mathrm{CO}_{3}\right)$ and dodecanethiol (DDT) were purchased from Aldrich and used without further purification. 2Methyl-2-oxazoline (MOXA) and acetonitrile were distilled over $\mathrm{CaH}_{2}$ just prior to use. Pheophorbide a (pheo) came from Wako Inc. Coumarin-functionalized methyl methacrylate (CmMMA) and coumarin tosylate were synthesized and purified by recrystallization according to already published protocols ${ }^{31}$ (figure S1 and S2). Acetonitrile and chloroform were distilled over $\mathrm{CaH}_{2}$ and stocked under nitrogen. Ultrapure water was obtained from an ELGA Purelab Flex system (resistivity higher than $18.2 \mathrm{M} \Omega . \mathrm{cm}$ ) and was filtered on $0.2 \mu \mathrm{m}$ RC filters just before use.

Typical cationic ring-opening polymerization of MOXA. The reaction was performed under nitrogen. The coumarin tosylate initiator ( $0.383 \mathrm{~g}, 0.78 \mathrm{mmol}$ ) and MOXA ( $1 \mathrm{~mL}, 11.8 \mathrm{mmol}$ ) were dissolved in $4 \mathrm{~mL}$ of anhydrous acetonitrile. The solution was stirred at $80{ }^{\circ} \mathrm{C}$ during 7 hours, followed by the addition of piperidine $(0.39$ $\mathrm{mL}, 3.92 \mathrm{mmol}$ ) at room temperature followed by $15 \mathrm{~h}$ stirring. The polymer was precipitated in cold diethylether $\left(0^{\circ} \mathrm{C}\right)$ and dried under vacuum. The product was obtained with a $69 \%$ yield.

CmPOX: ${ }^{1} \mathrm{H}$ NMR (400 MHz, $\mathrm{CDCl}_{3}$ ) (figure $\left.\mathrm{S} 2\right), \delta=7.4(\mathrm{~d}, 1 \mathrm{H}, \mathrm{H} 6)$, $6.8(\mathrm{~d}, 1 \mathrm{H}, \mathrm{H} 7), 6.7(\mathrm{~s}, 1 \mathrm{H}, \mathrm{H} 9), 6.1(\mathrm{~s}, 1 \mathrm{H}, \mathrm{H} 2), 3.9(\mathrm{~m}, 2 \mathrm{H}, \mathrm{H} 11), 3.7-$ $3.2(m,(4 n+2) H, H a), 2.4(s, 3 H, H 4), 2.4-2.1(m, 3 n, H b), 1.8-1.2$ (m, 18H, 13-21).

\section{$\mathrm{Mn}, 3140 \mathrm{~g} \mathrm{~mol}^{-1}, \mathrm{Mw}, 3840 \mathrm{~g} \mathrm{~mol}^{-1}$, Đ 1.22 (Figure S3).}

Formation of polymer self-assemblies. $20 \mathrm{mg}$ of polymer were dissolved in $2.5 \mathrm{~mL}$ of water. If needed, a small volume of additive acetone solution (12 - $15 \mu \mathrm{L}$ of CmMMA or CmPMMA solution, calculated to have a final desired ratio of either 6 or $13 \mathrm{wt} \%$ compared to (mPOX) was added under stirring at room temperature. The volume was calculated depending on the final desired ratio (typically 7 or 13 mol\% for CmPMMA and $1 / 30$ $\mathrm{mol} / \mathrm{mol}$ for Pheo). The solution was left standing for 2 days to evaporate acetone.

Coumarin dimerization. A 5-mm NMR tube containing $2.2 \mathrm{~mL}$ of the self-assembly solution was placed during 7 hours between two UV lamps (figures S4 and S5), Philips linear T5 8W, irradiation at 360 $\mathrm{nm}$, lamp-tube distance $8 \mathrm{~mm}$, total irradiance $1.0 \mathrm{~mW} . \mathrm{cm}^{-2}$, measured with a HD9021 photometer from Delta Ohm Inc.).

Polymerization of CmPOX/CmMMA micelles. The procedure was similar to already published procedures ${ }^{9}$. Typically, the micelle solution as prepared in the previous paragraph $\left(4 \mathrm{mg} \cdot \mathrm{mL}^{-1}\right)$ was purged with argon for 20 minutes and heated at $50^{\circ} \mathrm{C}$ for $48 \mathrm{~h}$.

CmMMA telomerization. The telomerization was carried out in acetonitrile using dodecanethiol as telogen agent with a 
DDT/CmMMA ratio at $0.1 \mathrm{~mol} / \mathrm{mol}$. CmMMA (2.59 g, $6.25 \mathrm{mmol}$ ) and DDT ( $0.15 \mathrm{~mL}, 0.625 \mathrm{mmol}$ ) were dissolved in $12 \mathrm{~mL}$ acetonitrile and the solution was purged with argon during 30 minutes. It was subsequently heated to $80^{\circ} \mathrm{C}$ and Azobisisobutyronitrile (AIBN) (5.0 $\mathrm{mg}, 0.031 \mathrm{mmol}$ ) dissolved in $0.125 \mathrm{~mL}$ acetonitrile was added. After 8 hours of reaction, a yield of $41 \%$ in telomer was recovered by precipitation in cold ethanol $\left(-18{ }^{\circ} \mathrm{C}\right)$, filtered and dried under vacuum.

CmPMMA: ${ }^{1} \mathrm{H}$ NMR $\left(400 \mathrm{MHz}, \mathrm{CDCl}_{3}\right), \delta=7.5(\mathrm{~d}, 1 \mathrm{H}, \mathrm{H} 6), 6.8(\mathrm{~d}, 1 \mathrm{H}$, $\mathrm{H} 7), 6.7(\mathrm{~s}, 1 \mathrm{H}, \mathrm{H} 9), 6.1(\mathrm{~s}, 1 \mathrm{H}, \mathrm{H} 2), 4.0(\mathrm{t}, 2 \mathrm{H}, \mathrm{H} 11), 3.6(\mathrm{t}, 2 \mathrm{H}, \mathrm{H} 21)$, $2.4(\mathrm{~s}, 3 \mathrm{H}, \mathrm{H} 4), 1.8-1.0(\mathrm{~m}, 16 \mathrm{H}+2 \mathrm{n}, \mathrm{H} 12-\mathrm{H} 20, \mathrm{HP} 23), 1.2(\mathrm{~m}, 3 \mathrm{n}$, HP22)

$\mathrm{Mn}=4300 \mathrm{~g} \cdot \mathrm{mol}^{-1} ; \mathrm{Mw} / \mathrm{Mn}=1.45$

Size Exclusion Chromatography. The SEC equipment was a Varian 390-LC model equipped with refractometric detector $(880 \mathrm{~nm})$. Two PL-gel mix C columns were used at $70{ }^{\circ} \mathrm{C}$ using $\mathrm{N}, \mathrm{N}$ dimethylformamide (DMF) ( $0.1 \mathrm{wt} \% \mathrm{LiBr}$ ) as eluent at a flow rate of $0.8 \mathrm{~mL}$. $\mathrm{min}^{-1}$. A (Poly(methyl methacrylate)) PMMA calibration was used to determine the average molar weights using PMMA standards from Agilent. Samples were injected at a $10 \mathrm{mg} \cdot \mathrm{mL}^{-1}$ concentration.

Encapsulation of Pheo. $2 \mu \mathrm{L}$ of a $1.2310^{-2} \mathrm{M}$ Pheo solution in acetone were added to $0.3 \mathrm{~mL}$ of a self-assembly aqueous solution $\left([\mathrm{CmPOX}]=2.5410^{-3} \mathrm{M}\right.$. The solution was left standing one night to evaporate acetone. A $1 / 30 \mathrm{~mol} / \mathrm{mol}$ Pheo/CmPOX was chosen based on previous experiments $\mathrm{s}^{8,9}$.

Dialyses experiments. Solutions of loaded Pheo/ self-assemblies were diluted in order to obtain a Pheo concentration at $10^{-6} \mathrm{M} .2 \mathrm{~mL}$ of these diluted solutions were introduced in a dialysis kit (GE Healthcare Bio-Sciences, membrane with MWCO at 8kDa) and dialyzed versus $800 \mathrm{~mL}$ of water at $37^{\circ} \mathrm{C}$. The release of Pheo was followed up by measuring the optical density of the internal solution at $688 \mathrm{~nm}$ (Pheo alone) and $669 \mathrm{~nm}$ (Pheo in selfassemblies). Each dialysis was performed twice. As comparison, Pheo release was also checked in PBS at $37^{\circ} \mathrm{C}$ in absence of vectors. This did not show any difference of behavior (data not shown), validating the use of pure water for all comparisons.

Dynamic light scattering (DLS). DLS was carried out at $25^{\circ} \mathrm{C}$ on a Malvern (Orsay, France) Zetasizer NanozS. Solutions were analyzed in triplicate without being filtered in order to characterize the plain samples. Data were analyzed by the general-purpose non-negative least squares (NNLS) method. The typical accuracy for these measurements was $10-20 \%$ for systems exhibiting a polydispersity index lower than 0.4. In the case of cross-linked CmPOX/CmPMMA samples, multiangle dynamic light scattering was performed with a LS Spectrometer (LSinstruments) in 3D configuration measuring the scattering from $20^{\circ}$ to $150^{\circ}$ each $5^{\circ}$ or $2^{\circ}$.

All correlograms were analyzed by a custom-made program named STORMS in order to obtain a more precise characterization of the solutions ${ }^{34}$. This program has been designed with Matlab and enables the fitting of DLS correlograms using different sets of parameters, corresponding to all hypotheses that have to be made during the treatment. Indeed, going from correlograms to size results implies three levels of hypotheses: the first one consisting in the transformation of autocorrelation data to diffusion coefficient, the second one extracting the size of the scattering object from diffusion coefficient depending on its geometry, and finally using a model enabling the transformation of the intensity-relative population to a number-relative one. For each step, STORMS provides the choice of different parameters. For the nano-objects presented here, the protocol used a NNLS fitting, assumed a spherical shape for all objects, and the chosen scattering model was that corresponding to a mixture of micelles and vesicles (maximum micelle size fixed at a radius of $25 \mathrm{~nm}$ ). Different sets of the range of decay rates and the regularization parameter were used, $\alpha=2$, range $=2$ being the most appropriate one for the samples of this work. Unless stated, this treatment provided residuals lower than $5 \times 10^{-3}$ for all analyses. The polydispersity index (PDI) is the ratio between the variance of the distribution and the square of the mean value of the decay rate, $\overline{\boldsymbol{\Gamma}}$. When the autocorrelation functions were registered at different angles, the $\overline{\boldsymbol{\Gamma}}$ obtained is plotted as a function of $\mathrm{q}^{2}$ in order to obtain the mean diffusion coefficient and, from Stokes-Einstein equation, the mean hydrodynamic radius according to equation $\bar{\Gamma}=\boldsymbol{D} q^{2}$.

For the evaluation of DMF resistance, similar DLS experiments were performed with increasing quantities of DMF. The position of the measurements was fixed and the refractive index was changed depending on the DMF quantity.

Transmission Electron Microscopy (TEM). TEM analyses were performed with a Hitachi HT7700 (Hitachi High Tech, Hitachinaka, Japon) microscope (accelerating voltage of $75 \mathrm{kV}$ ). Small amounts of particle suspensions in water were deposited onto a discharged copper grid coated with a carbon membrane, left for 1-3 min depending on the solution, and gently dried with absorbing paper. A drop of uranyl acetate solution was deposited onto the grid for 10 seconds, and the grid was then dried under a lamp for at least 5 $\min$. When the images contained a large number of distinct objects (typically > 200), a measurement of the mean size (as well as the standard deviation) was performed with Image J software (http://imagej.nih.gov/ij/).

TEM analyses were also performed one month after the formation of the nanovectors and showed no evolution, proving that either crosslinked or uncrosslinked CmPOX/CmPMMA vectors were at least stable one month in pure water at room temperature.

Cryo-Electron Microscopy (Cryo-TEM). $3 \mu \mathrm{L}$ of sample were deposited onto glow-discharged lacey carbon grids and placed in the thermostatic chamber of a Leica EM-GP automatic plunge freezer, set at $20^{\circ} \mathrm{C}$ and $95 \%$ humidity. Excess solution was removed by blotting with Whatman $\mathrm{n}^{\circ} 1$ filter paper for 1,2 seconds, and the grids were immediately flash frozen in liquid ethane at $-185^{\circ} \mathrm{C}$. The frozen specimens were placed in a Gatan 626 cryo-holder, and cryo TEM was carried out on a Jeol 2100 microscope, equipped with a $\mathrm{LaB}_{6}$ cathode and operating at $200 \mathrm{kV}$, under low dose conditions. Images were acquired with SerialEM software, with defocus of 1.5$3 \mu \mathrm{m}$, on a Gatan US4000 CCD camera. This device was placed at the end of a GIF Quantum energy filter (Gatan, Inc.), operated in zero-energy-loss mode, with a slit width of $25 \mathrm{eV}$. Images were 
recorded at a nominal magnification of 4,000 corresponding to calibrated pixel sizes of $1.71 \AA ̊$.

Cell culture. The HCT-116 cell line (ATCC \#CCL-247) originated from a human colorectal carcinoma. HCT-116 cells were grown in Dulbecco's Modified Eagles Medium (Invitrogen) containing glucose $(4.5 \mathrm{~g} / \mathrm{L})$, GlutaMAX and pyruvate, supplemented with $10 \%(\mathrm{v} / \mathrm{v})$ heat inactivated fetal bovine serum (FBS), $100 \mathrm{U} / \mathrm{mL}$ penicillin and $100 \mu \mathrm{g} / \mathrm{mL}$ streptomycin. Cells were maintained at $37^{\circ} \mathrm{C}$ in a humidified atmosphere containing $5 \% \mathrm{CO}_{2}$.

Cytotoxicity and phototoxicity of Pheo-loaded polymeric nanovectors. These biological tests were performed on 2D adherent cell monolayers. HCT-116 tumor cells were seeded in 96well plates $(4,000$ cells per well) $24 \mathrm{~h}$ prior to experiment. Cytotoxicity of polymer self-assemblies was assessed after $24 \mathrm{~h}$ of cells incubation with nanovectors at a CmPOX polymer concentration of $30 \mu \mathrm{M}$. To assess phototoxicity of nanovectors in a photodynamic therapy context, cells were incubated for $30 \mathrm{~min}$ at $37^{\circ} \mathrm{C}$ with Pheo-loaded self-assemblies $(1 / 30 \mathrm{~mol} / \mathrm{mol}$ ) (i.e $1 \mu \mathrm{M}$ of pheophorbide). Then, cells underwent a set of two photoactivations 8 , which were performed with an overhead projector with a bandpass filter $(\lambda>400 \mathrm{~nm})$, representing a total dose of $12 \mathrm{~J} \mathrm{~cm}^{-2}$. In brief, cells were illuminated for 2 minutes, followed by 2 minutes off, and once again 2 minutes of illumination. Concentration used for Pheo alone was $1 \mu \mathrm{M}$ corresponding to the same concentrations as in the experiments with the loaded polymer self-assemblies. Viability was assessed using a metabolic test called PrestoBlue reagent (Invitrogen) according to the manufacturer instructions. For every set of experiments led three times independently, six biological replicates were produced and analyzed. Statistical differences between values were assessed by one-way ANOVA followed by a Dunnett's multiple comparisons test which compare each condition with control condition. All data were expressed as mean \pm standard error of the mean (SEM), and overall statistical significance was set at $p<0.05$. A set of PDT experiments was led independently two times with 0.1 and $0.5 \mu \mathrm{M}$ pheo-loaded nanovectors.

\section{Results}

In order to obtain a polymer micelle, poly(2-methyl-2-oxazoline) (POX) was employed as hydrophilic stabilizing external block. The hydrophobic core was composed of a C11 alkyl chain to help the self-assembling process, both connected to POx block and a photoreactive group such as coumarin, well-known to dimerize under UV irradiation, as already published $^{31}$ (Figure S1). This linear amphiphilic polymer (CmPOX) permits only to photo-link two by two the tangled chains but not crosslink the micelle core as previously discussed in a comparison to grafted amphiphilic copolymers ${ }^{35}$. To ensure the stabilization of the micelle by crosslinking, a coumarin-functionalized methyl methacrylate $(\mathrm{CmMMA})^{36}$ was added (Scheme 1, step 2a) since it was able to react by photo-dimerization both with its own coumarin unit as well as that of CmPOX (Scheme 1, step 3a) and photo-polymerization of the methacrylate unit. 


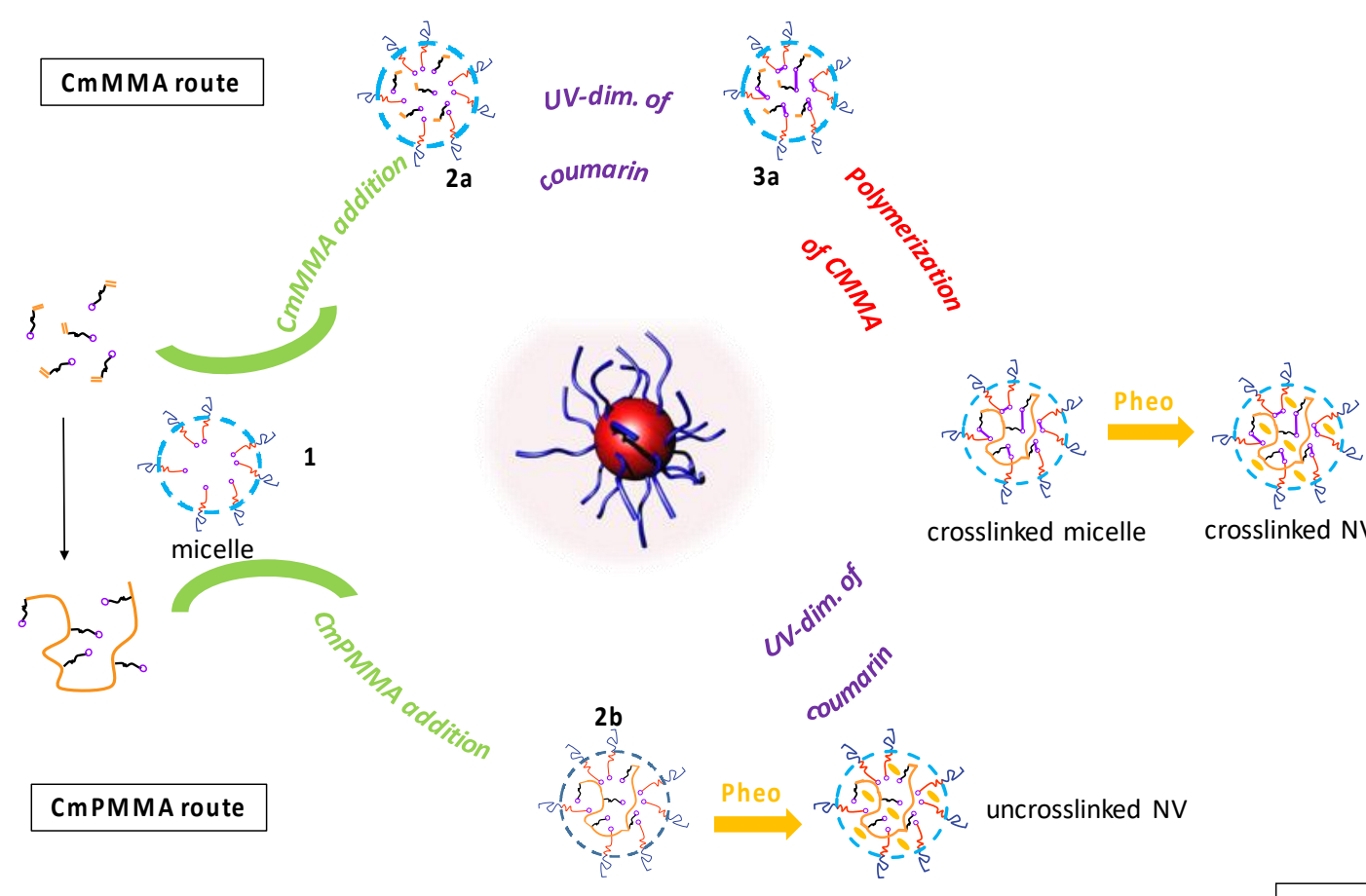

$\curvearrowleft$ dimerized coumarin
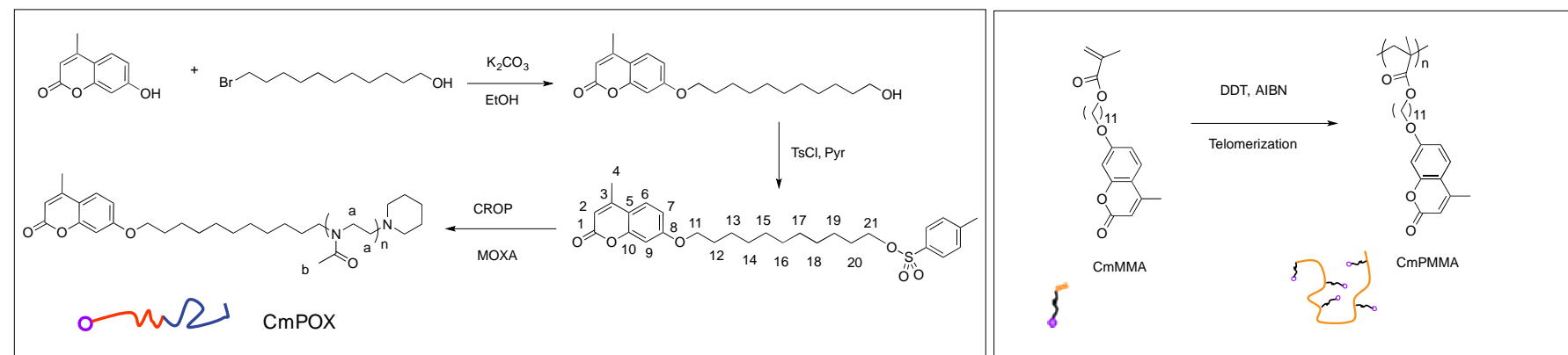

Scheme 1. Principle of formation of photo-crosslinked micelles.

CmPOX was directly dispersed in water and small $13-\mathrm{nm}$ polymer micelles were observed by cryo-TEM (Scheme 1, step 1, figure S10), which was consistent with earlier results ${ }^{36}$. CmMMA was then encapsulated into the formed micelles by addition of a small volume of CmMMA acetone solution (Scheme 1, step 2a). A CmMMA /CmPOX ratio at 7 mol\% (1 wt \%) was chosen to avoid any destabilization of the micelles. This was checked by TEM images, and showed that no modification was present even after subsequent coumarin dimerization and CmMMA radical polymerization (Figure S6). The monitoring of the polymerization by ${ }^{1} \mathrm{H}$ NMR spectroscopy (Figure S7) showed an incomplete polymerization leading to the presence of residual monomer. Since its uncontrolled release could have been detrimental for the PDT application, this path was abandoned.

In order to avoid the residual CmMMA into the self-assemblies, this monomer was first polymerized before encapsulating into the CmPOX micelles (Scheme 1, CmPMMA route). Telomerization was performed using dodecanethiol as transfer agent and AIBN as radical initiator following well-known procedures ${ }^{37}$. The resulting CmPMMA oligomers were characterized by ${ }^{1} \mathrm{H}$ NMR and Size Exclusion Chromatography (Figures S8 and S9) and exhibited a molar mass close to $4000 \mathrm{~g}^{\mathrm{mol}}{ }^{-1}$. Its encapsulation in CmPOX micelles was similarly performed as CmMMA route using a small acetone solution volume on preformed CMPOX micelles (Scheme 1, step $2 \mathrm{~b}$ ). In this system, the amount of encapsulated CmPMMA was $13 \mathrm{wt} \%$, which showed by TEM the presence of the same type of 
micelles compared to $\mathrm{CmPOX}$ alone or $\mathrm{CmPOX} / \mathrm{CmMMA}$ (Figure S10).

The mixed micelles were then irradiated at $360 \mathrm{~nm}$ to crosslink their hydrophobic core by cross- and sym-dimerization between coumarin units of CMPOx and CMPMMA. TEM analyses showed small micelles of $\mathrm{CmPOx}$ close to $10 \mathrm{~nm}$ which did not change with the addition of CmPMMA and upon the irradiation and the loading in Pheo (Figure 1). It is noteworthy that TEM images of Pheo-loaded nanovectors were very different from Pheo dispersed in water (Figure S11). Cryo-TEM characterization confirmed the presence of small micelles exhibiting a size between 13 and $26 \mathrm{~nm}$ (Figure 1). Although TEM and cryo-TEM enable to visualize the self-assemblies, they both have well-known limitations. TEM implies drying of the sample which might lead to a re-organization while cryo-TEM can enrich the solution in small objects owing to the very thin ice thickness. Furthermore, they both provide the analysis of only several hundreds of objects outmost, which might not reflect the overall sample solution.
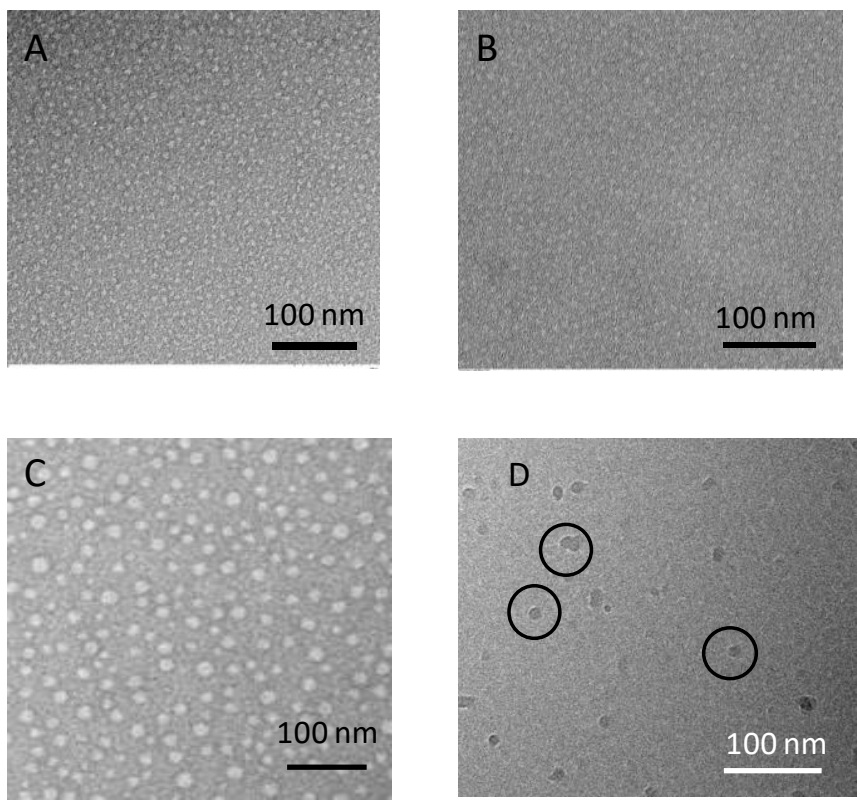

Figure 1. TEM images of CmPOX/CmPMMA micelles: uncrosslinked (A), crosslinked (B) and crosslinked and loaded with Pheo (C). CryoTEM images of crosslinked CMPOX/CmPMMA micelles loaded in Pheo (D)

Therefore, the second typical characterization of self-assembly size is mono-angle Dynamic Light Scattering (DLS), which enables the batch analysis of the solution. The intensity-average analysis showed the presence of large objects, with a first population close to $300 \mathrm{~nm}$ and a second one exhibiting a size above $600 \mathrm{~nm}$ (Figures 2 and S12). On the other hand, the number average analysis pointed to the majority presence of small micelles with a size lower than $50 \mathrm{~nm}$. However, the presence of large objects impeded any detailed characterization of this population. This result can be explained taking into account the scattered light intensity dependency with the size of the scattering object. Since, this is linked to $R^{6}$, it is well known that the presence of even a small amount of large objects will hinder the DLS detection of small ones $^{34}$. This can be considered as an extreme case where the difference of small versus large self-assemblies is too large to properly extract the exact size of the micelles by DLS. However, DLS pointed to the presence of large objects. These were indeed observed by TEM, but on very few images (Figure S13).

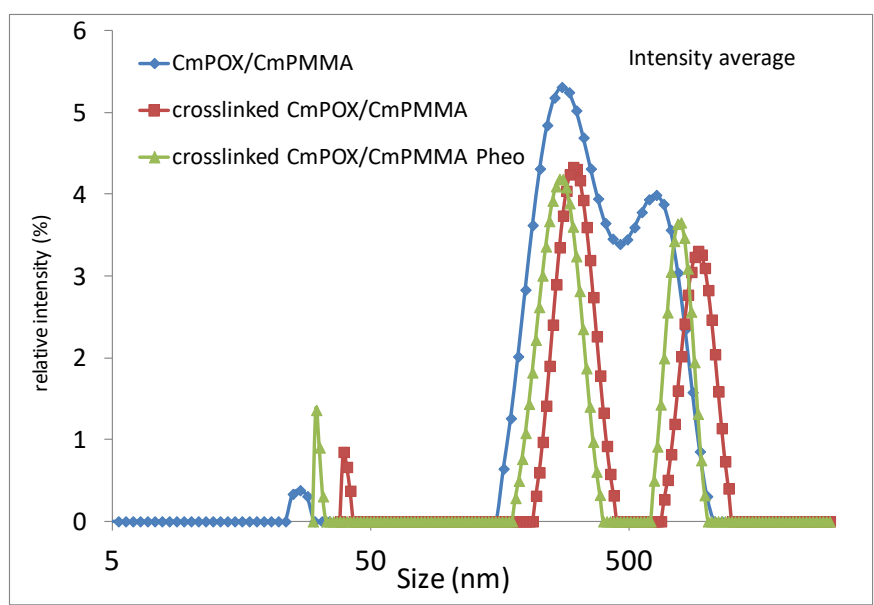

Figure 2. Intensity-average DLS of CmPOX/CmPMMA selfassemblies.

In order to analyze more thoroughly the sample solutions, further characterization was carried out by multi-angle DLS in the case of crosslinked CmPOX/CmPMMA (figures S14 and S15). The direct analysis of the solution yielded the same result than the monoangle DLS characterization, i.e. the presence of large objects with a size close to $280 \mathrm{~nm}$ in good agreement with the smaller population observed at $173^{\circ}$ (see DLS Int in Table 1). It is noteworthy that these analyses were performed after dilution of the samples and that no population at ca. $950 \mathrm{~nm}$ was observed contrarily to mono-angle DLS. This points to the hypothesis that this $950 \mathrm{~nm}$ population is constituted of self-assemblies aggregates which were destroyed by dilution. Filtration of the solution at $0.2 \mu \mathrm{m}$ was performed in order to eliminate the large objects and allowing a better estimation of the size of the smaller self-assemblies. This led to the appearance of 2 populations below $100 \mathrm{~nm}$, the first one close to $90 \mathrm{~nm}$ and the second one around $6 \mathrm{~nm}$ in accordance with the more abundant population observed by TEM. Comparing the scattered intensity before and after filtration indicated that the small populations concentrations were at least 30 times higher than the $280 \mathrm{~nm}$ objects.

Regarding the overall characterization of these self-assemblies, this is an extreme case illustrating the importance of multiple analyses of polymer self-assemblies. Based on Table 1 the main conclusions are that the majority of self-assemblies exhibits a size below $40 \mathrm{~nm}$ whereas a minority has a size above $200 \mathrm{~nm}$. The quantification of each population and their further characterization will imply the use of separation techniques such as Flow Field Flow Fractionation, as well as other scattering techniques such as X-ray or neutron scattering. This was however beyond the scope of this study.

As already discussed, the development of crosslinked polymeric vectors aims at increasing their stability. It is therefore important to examine the resistance of such objects towards challenging 
conditions. Before confronting them to biological media, a useful test is to assess their resistance towards a solvent which is able to dissolve the copolymers. For this, uncrosslinked and crosslinked CMPOX/CmPMMA solutions were diluted to increasing amount of DMF and characterized by single-angle DLS (Table 2).

\begin{tabular}{cccc}
\hline DCRth & 39200 & 29400 & 19600 \\
DCRexp/DCRth & 1.10 & 0.84 & 0.53 \\
\hline $\begin{array}{c}\text { Uncrosslinked } \\
\text { CmPOX/CmPMMA } \\
\text { DCRexp }\end{array}$ & 26700 & 10900 & 4700 \\
DCR th & 41400 & 31100 & 20700 \\
DCRexp/DCRth & 0.64 & 0.35 & 0.23 \\
\hline
\end{tabular}

Table 1. Polymer self-assemblies characterization

\begin{tabular}{cccccc}
\hline Technique & CmPOX & $\begin{array}{c}\text { CmPOX/ } \\
\text { CmPMMA }\end{array}$ & $\begin{array}{c}\text { Crosslinked } \\
\text { CmPOX/ } \\
\text { CmPMMA }\end{array}$ & $\begin{array}{c}\text { CmPOX/ } \\
\text { CmPMMA } \\
/ \text { Pheo }\end{array}$ & $\begin{array}{c}\text { Crosslinked } \\
\text { CmPOX/ }\end{array}$ \\
$\begin{array}{cccccc}\text { CmPMMA } \\
/ \text { Pheo }\end{array}$ \\
\hline DLS Int & -a & $280 / 612$ & $320 / 950$ & - a & $278 / 780$ \\
DLS Num & & 244 & 41 & & 32 \\
TEM & & $9 \pm 2$ & $8 \pm 2$ & n.d. & $11 \pm 2$ \\
Cryo-TEM & $13.2 \pm$ & n.d. & n.d. & $\begin{array}{c}15.9 \pm 2.5 \\
\text { b }\end{array}$ & $12.7 \pm 3.5$ \\
\hline & 2.5 & & & & \\
\hline
\end{tabular}

a DLS data not reliable enough for treatment. ${ }^{\mathrm{b}}$ number of objects measured $<30$.

The characterization of the self-assemblies by TEM proved that Pheo was very probably inside the polymer micelles and that loading did not modify their size. A further proof was given by UVvisible absorption spectra, particularly zooming on the $600-700$ $\mathrm{nm}$ area. Indeed, it is well-known that Pheo absorbance is sensitive to its environment. In aqueous conditions, the band of this zone exhibits a maximum at $690 \mathrm{~nm}$ (Figure 3). In the presence of crosslinked CmPOX/CmPMMA micelles, this band was shifted to $670 \mathrm{~nm}$, which is characteristic of a more hydrophobic environment ${ }^{7}$ such as the micelle core composed of alkyl chains decorated by coumarin dyes.

Since we formerly discussed the problem of evaluating the size of the objects by DLS, we only focused on the scattered light intensity (as evaluated through Derived Count Rate, DCR). This is furthermore relevant information to assess, since presenting only the value of the size obtained by DLS might be misleading ( $a$ mixture of $95 w t \%$ free disintegrated polymer/ $5 w t \%$ of nano-object would exhibit the same un-modified DLS result compared to the pure nano-object). The experimental DCR was compared to the theoretical minimum one, obtained by a simple decrease of the intensity owing to dilution, therefore not taking into account possible swelling upon presence of the good solvent. Any strong decrease compared to this theoretical DCR should be a sign of selfassembly disintegration owing to dissolution. In such test, uncrosslinked systems are expected to resist only poorly to the addition of a good solvent, whereas crosslinked ones should possibly swell without disintegrating. Table 2 shows that it is indeed the case, with the uncrosslinked system beginning to dissociate at 20 vol\% of DMF, whereas the crosslinked one holds up to 40 vol\%. This result is very comparable to other studies presenting the scattered intensity 38,39 . Soft nano-objects like these polymeric micelles, although crosslinked, remain breakable under harsh conditions such as this exposure to good solvent. In fact, among all crosslinked polymeric micelles described in the literature, only one example has been shown to exhibit an ideal behavior, remaining completely stable upon organic solvent addition ${ }^{40}$.

Table 2. Swelling test in DMF of various CmPOX/CmPMMA micelles monitored by scattered intensity.

\begin{tabular}{ccccc}
\hline & 0\% DMF & 20 vol\% & 40 vol\% & 60 vol\% \\
\hline $\begin{array}{c}\text { Crosslinked } \\
\text { CmPOX/CmPMMA } \\
\text { DCRexp }\end{array}$ & 49000 & 41700 & 24700 & 10300 \\
\hline
\end{tabular}

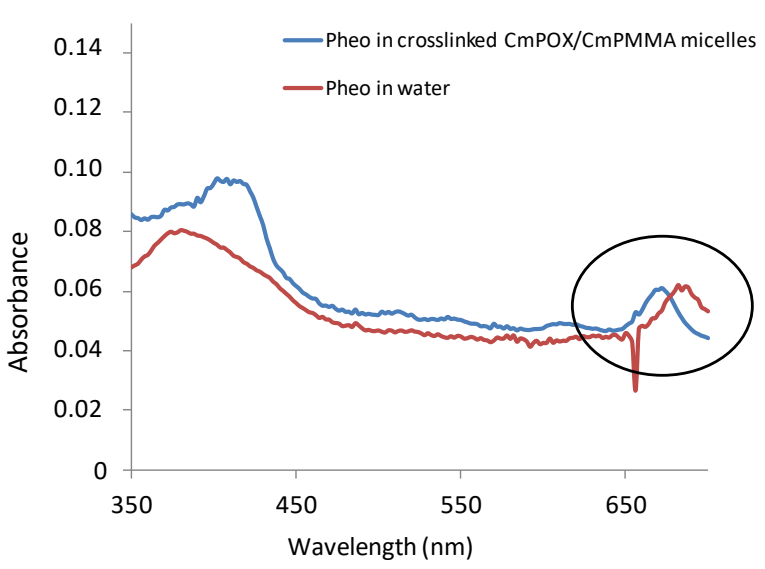

Figure 3. UV-visible absorption of Pheo in water or in CmPOX/CmPMMA crosslinked systems.

Before assessing cytotoxicity and PDT efficiency of these polymeric nanovectors, a last physico-chemical characterization consisted in examining the kinetics of Pheo release in simple dialysis models as illustrated in Figure 4. The results show that Pheo was released more slowly in crosslinked nanovectors compared to the uncrosslinked ones. For uncrosslinked vectors, Pheo was released in a similar manner than Pheo alone (in the absence of any vector) at the beginning of the dialysis. 


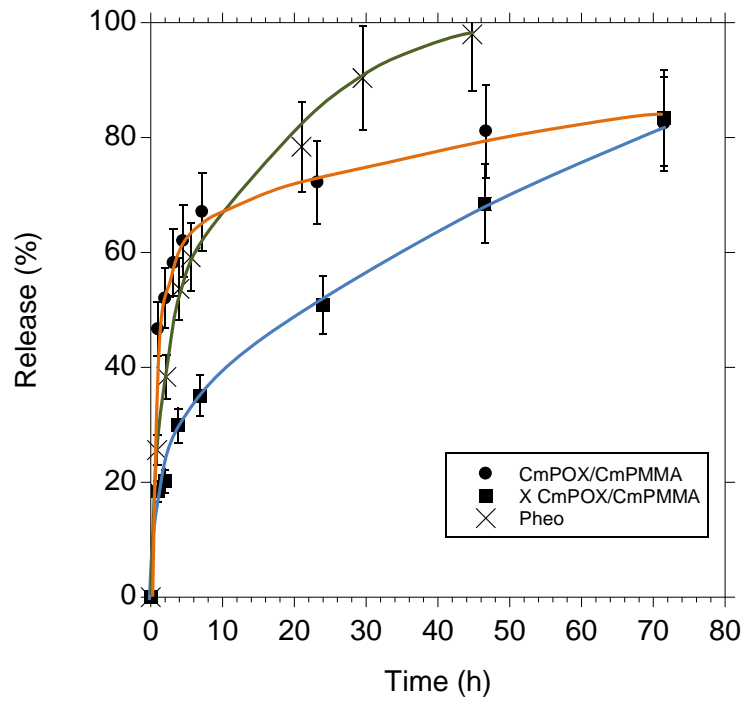

Figure 4. Pheo release follow-up by dialysis. [Pheo] $=10^{-6} \mathrm{M}, 37^{\circ} \mathrm{C}$, in pure water).

After physico-chemical characterization of these nanovectors, we moved to biological tests. Three distinct in vitro experiments experiments on human colorectal cancer cells HCT-116 showed that no significant cell viability decrease was observed after $24 \mathrm{~h}$ of incubation with the first generation of micelle based on CmPOX or CMPMMA alone (Figure 5A). However, it has to be noted that micelles bearing in its core CmPMMA statistically decrease cell viability in uncrosslinked and tends to decrease cell viability in the crosslinked condition $(86.6 \%$ and $89.2 \%$ respectively).It is noteworthy that Pheo alone is known to be non cytotoxic without any irradiation in the same concentration 8,9,34.

PDT experiments were led in vitro on human tumor cells HCT-116 to assess the potential of these nanovectors loaded or not with $1 \mu \mathrm{M}$ Pheo for photodynamic therapy as summarized in Figure 5B. Nonpheo loaded CmPOX, CmPMMA, un-crosslinked (CMPOX/CmPMMA) and crosslinked (X CmPOX/CmPMMA) nanovectors did not alter cell viability. At this $1 \mu \mathrm{M}$ concentration, pheo alone statistically reduced cell viability by approximately $7 \%$, which was consistent with already published results ${ }^{9}$. Interestingly, when this photosensitizer was encapsulated in un-crosslinked or crosslinked nanovectors, cell viability dramatically decreased to only $30 \%$. This means that nanovectors were highly efficient to deliver Pheophorbide a to tumor cells. It has to be noted that the difference observed between uncrosslinked and crosslinked nanovectors was not statistically significant, which means that crosslinking did not bring a supplementary beneficial effect of nanovectors in terms of photodynamic therapy in this monolayer cell culture. Other PDT experiments were led with lower pheophorbide a concentration (i.e $0.1 \mu \mathrm{M}$ and $0.5 \mu \mathrm{M}$ ) (Figure S16). A dose effect was observed: when encapsulated in un-crosslinked (CmPOX/CmPMMA) and crosslinked ( $\mathrm{X}$ CmPOX/CmPMMA) nanovectors, cell viability was quantified around $65 \%$ with $0.1 \mu \mathrm{M}$ Pheo-loaded and $30 \%$ with
$0.5 \mu \mathrm{M}$ pheo-loaded. Interestingly, in these conditions, pheophorbide alone was not concentrated enough to induce a loss of cell viability, indicating a high benefit to encapsulate it within nanovectors.

\section{Discussion}

The ideal nanovector should exhibit numerous properties, such as adequate size to benefit from EPR effect, surface zeta potential close to neutrality, biocompatibility, delivery of its cargo at an appropriate rate depending on the application. To optimize the vectors and get closer to this difficult goal, several leads have been examined in the literature, one of these being crosslinking the vector, increasing its intrinsic stability and therefore its mean blood circulation time. The same increase of stability has also been observed in the vector presented here, since its resistance to swelling in DMF has been improved substantially as shown by the scattered light intensity which did not drop drastically before 60 vol\% of DMF.

Developing polymeric nanovectors implies thorough characterization of the self-assemblies, which can be often tricky, owing to the possible presence of multi-populations. The nanovector presented here is a typical difficult case. Indeed, both TEM and cryo-TEM indicated the presence of small nano-objects below $20 \mathrm{~nm}$, whereas the classically used intensity-average DLS analysis ranged from $200 \mathrm{~nm}$ to $1 \mu \mathrm{m}$. This discrepancy can be explained by the intrinsic weakness of DLS in analyzing multipopulation systems, as we clearly showed in a previous study ${ }^{34}$. Using multi-angle static/dynamic light scattering proved unsuccessful in resolving this situation. Nanoparticle Tracking Analysis (NTA) is another technique often used to characterize vesicles, providing both their size and the distribution of different populations. NTA was used for the system developed here (data not shown), but, unsurprisingly, only the large population was once again observed, since NTA is known for its lower size limit, close to $50 \mathrm{~nm}$ for soft matter. Another possible technique is Flow Field Flow Fractionation (FIFFIF), providing a separation of the nanoobjects by their size before analysis by light scattering detectors. We have previously shown that this technique is particularly relevant for the characterization of nanovectors, such as poly(ethylene oxide-b- $\varepsilon$-caprolactone) ones ${ }^{41,}{ }^{42}$. Tentative analysis of the present samples was performed, but led to unreproducible results, possibly owing to responsiveness of the vectors to the shear in the FIFFIF cell, however small. Developing an appropriate method to characterize CmPOX self-assemblies was beyond the scope of this work, but should be performed before any in vivo injection, to get a precise knowledge of the vector solution content. 
A

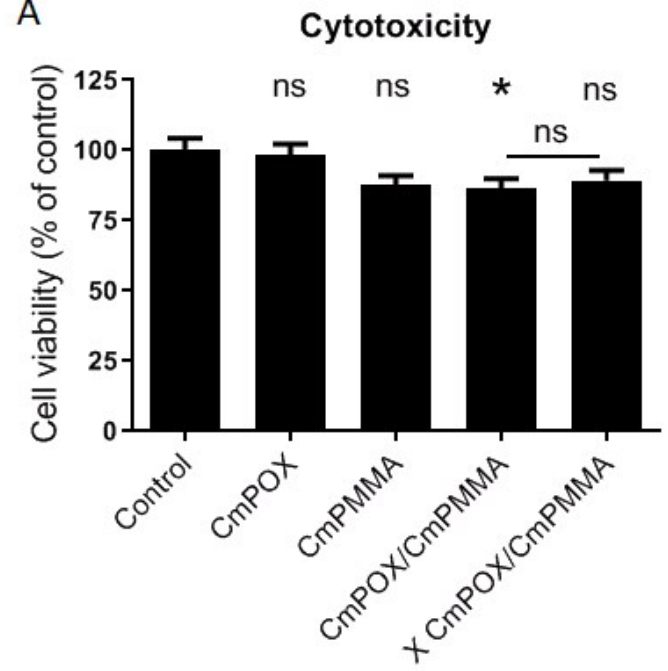

B

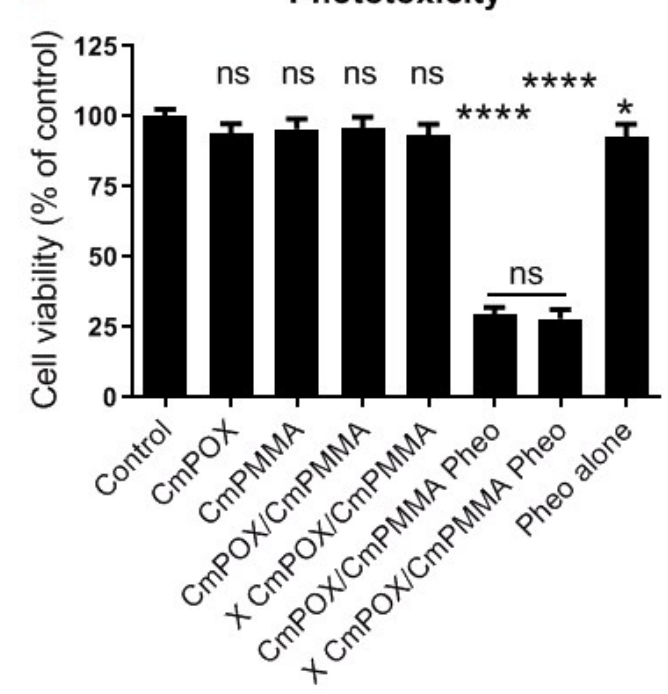

Figure 5. Cytotoxicity and phototoxicity of (un)crosslinked polymeric nanovectors (CmPOX/CmPMMA) on human tumor cells HCT-116. A. Cytotoxicity was quantified with the metabolic test PrestoBlue after $24 \mathrm{~h}$ of incubation with (un)crosslinked polymeric nanovectors CmPOX/CmPMMA. B. Efficacy of $1 \mu \mathrm{M}$ Pheo-loaded nanovectors on cell viability after photodynamic therapy protocol assessed with the metabolic test PrestoBlue $24 \mathrm{~h}$ after treatment. $\mathrm{X}$ : crosslinked nanovector; Pheo: pheophorbide a. Statistical analysis was led by one-way ANOVA followed by a Dunnett's multiple comparisons test on 18 independent biological replicates comparing each condition with the control one. Statistical significance was compared between pheo-loaded crosslinked and non-crosslinked nanovector using t-test.. $\mathrm{p}$ value $<0.05=*$; $\mathrm{p}<0.0001=* * * ; \mathrm{ns}=$ non-significant.

Coumarin was chosen in the process for its ability to dimerize under UV irradiation, enabling crosslinking of the constructions. However, coumarin ring system is well known to display multiple in vitro, in vivo and ex vivo pharmacological effects such as antimitotic, anticancer and cytotoxic effects ${ }^{43,44}$. For that purpose, we analyzed nanovector cytotoxic effect on human tumor colorectal cancer cells HCT-116. Interestingly, once integrated in the nanovectors, coumarin did not interfere with cell viability (CMPOX or CmPMMA) but when combined in CmPOX/CmPMMA nanovectors presenting higher concentration of coumarin, a slight trend was observed in decreasing cell viability. This property is interesting because it reinforces the anticancer potential of the nanovectors designed in this study for photodynamic therapy. In the literature, only a few cases of polymeric self-assemblies crosslinked through coumarin dimerization have been described. One example was from our team using different polyoxazoline polymers ${ }^{31,35}$, 45. This was focused on the physical chemistry aspect, in order to better understand how the microstructure of the polymer could interfere with the stability of the self-assemblies. Two other studies described coumarincrosslinked polymeric nanovectors for the release of doxorubicin ${ }^{46}$, 47 , either for a light-induced release or as simple photocrosslinkable system. Owing to the already mentioned biological properties of coumarin, such studies have to be controlled so that coumarin behavior is unambiguous. In the case of this study, the biological tests prove that the main response comes from the PDT effect coming from Pheo.

We also showed that polymeric self-assemblies, both un-crosslinked and crosslinked, drastically decreased tumor cell viability after photo-activation. These results are comparable with other systems previously described by our group 7,8 . We previously demonstrated with poly(ethylene oxide-b- $\varepsilon$-caprolactone) systems in 2D cell cultures that crosslinked and un-crosslinked vectors were efficient in reducing cell viability ${ }^{9}$. In the literature, crosslinking of the nanovectors is most often performed to optimize in vivo resistance. Therapeutic efficiency is not systematically improved, and one should be very careful in commenting $2 \mathrm{D}$ in vitro results. Indeed, examples have shown that in some cases crosslinked vectors are less efficient compared to free drug in vitro but this is reversed in vivo ${ }^{48-50}$. Avoiding systematic in vivo experiments is important for ethics, and it is the reason why synthetic 3D cell models have been increasingly developed. Thus, in our earlier study ${ }^{9}$, we also showed in a 3D tumor model named spheroid that the crosslinked vectors presented higher antitumor activity than un-crosslinked ones. Such a behavior was also observed by Stenzel using vectorized paclitaxel51,52. A way to go beyond in deciphering potential in photodynamic therapy would be to analyze interactions and efficiency of these nanoparticles with tumor and normal cells grown in a $3 \mathrm{D}$ tissue model such as spheroids.

Finally, comparing our results with those obtained on poly(2-ethyl-oxazoline-b-D,L-lactide) by Lai shows close results. Lai determined an IC50 for their photosensitizer close to $1.5 \mu \mathrm{M}$ whereas our system is between 0.1 and $0.5 \mu \mathrm{M}$. However, one should be very careful in the PDT comparison, since the cell lines are different, as well as the exact protocol of photosensitizer irradiation (power, duration...). A strong difference is however that contrary to them, we observe a strong improvement of the in vitro PDT 
treatment by encapsulation of the chosen photosensitizer, underlining the importance of the design of the nanovector.Conclusion

The efficiency of poly(2-methyl-2-oxazoline) self-assemblies as potential Pheophorbide a carrier for photodynamic therapy was demonstrated. Pheo has been undeniably encapsulated in the self-assemblies increasing its photocytotoxicity. Consequently POX polymers constitute a powerful platform for further development of nanovectors. The introduction of a controlled and limited aliphatic chain and the photoreactive coumarin units provides parameters to tune finely the vectors. Further work will be performed in order to adapt the vector to in vivo conditions.

\section{Conflicts of interest}

There are no conflicts to declare.

\section{Acknowledgements}

\section{Notes and references}

1. V. Weissig and D. Guzman-Villanueva, Int. J. Nanomedicine, 2015, 10, 1245-1257.

2. Z. Tang, C. He, H. Tian, J. Ding, B. S. Hsiao, B. Chu and X. Chen, Prog. Polym. Sci., 2016, 60, 86-128.

3. A. Varela-Moreira, Y. Shi, M. H. A. M. Fens, T. Lammers, W. E. Hennink and R. M. Schiffelers, Materials Chemistry Frontiers, 2017, 1, 1485-1501.

4. Y. Matsumura and H. Maeda, Cancer Research, 1986, 46, 6387-6392.

5. H. Maeda, Bioconjugate chem., 2010, 21, 797-802.

6. S. S. Lucky, K. C. Soo and Y. Zhang, Chem. Rev., 2015, 115, 1990-2042.

7. K. Knop, A.-F. Mingotaud, N. El-Akra, F. Violleau and J.-P. Souchard, Photochem. Photobiol. Sci., 2009, 8, 396-404.

8. L. Gibot, A. Lemelle, U. Till, B. Moukarzel, A. F. Mingotaud, V. Pimienta, P. Saint-Aguet, M. P. Rols, M. Gaucher, F. Violleau, C. Chassenieux and P. Vicendo, Biomacromolecules, 2014, 15, 1443-1455.

9. U. Till, L. Gibot, P. Vicendo, M. P. Rols, G. Gaucher, F. Violleau and A. F. Mingotaud, RSC Advances, 2016, 6, 69984-69998.

10. S. Wang, F. Yuan, K. Chen, G. Chen, K. Tu, H. Wang and L.-Q. Wang, Biomacromolecules, 2015, 16, 2693-2700.

11. H. Hu, X. Yan, H. Wang, J. Tanaka, M. Wang, W. You and Z. Li, J. Mater. Chem. B, 2019, 7, 1116-1123.

12. Y. Han, Z. Chen, H. Zhao, Z. Zha, W. Ke, Y. Wang and Z. Ge, J. Controlled Release, 2018, 284, 15-25.

13. Y. Wang, G. Wei, X. Zhang, F. Xu, X. Xiong and S. Zhou, Advanced Materials, 2017, 29, 1605357.

14. X. Wei, L. Liu, X. Guo, Y. Wang, J. Zhao and S. Zhou, ACS Applied Materials \& Interfaces, 2018, 10, 17672-17684.
15. X. Wang, J. Wang, J. Li, H. Huang, X. Sun and Y. Lv, J. Drug Delivery Science and Technology, 2018, 48, 414-421.

16. K. Knop, R. Hoogenboom, D. Fischer and U. S. Schubert, Angew. Chem. Int. Edn, 2010, 49, 6288-6308.

17. H. Schellekens, W. E. Hennink and V. Brinks, Pharm. Res., 2013, 30, 1729-1734.

18. Q. Yang and S. K. Lai, Wiley Interdisciplinary Reviews: Nanomedicine and Nanobiotechnology, 2015, 7, 655-677.

19. A. S. Abu Lila, H. Kiwada and T. Ishida, J. Controlled Release, 2013, 172, 38-47.

20. O. Sedlacek, B. D. Monnery, S. K. Filippov, R. Hoogenboom and M. Hruby, Macromol. Rapid Comm., 2012, 33, 16481662.

21. T. Lorson, M. M. Lübtow, E. Wegener, M. S. Haider, S. Borova, D. Nahm, R. Jordan, M. Sokolski-Papkov, A. V. Kabanov and R. Luxenhofer, Biomaterials, 2018, 178, 204280.

22. T. R. Dargaville, J.-R. Park and R. Hoogenboom, Macromol. Biosci., 2018, 18, 1800070.

23. P. H. Kierstead, H. Okochi, V. J. Venditto, T. C. Chuong, S. Kivimae, J. M. J. Fréchet and F. C. Szoka, J. Controlled Release, 2015, 213, 1-9.

24. R. W. Moreadith, T. X. Viegas, M. D. Bentley, J. M. Harris, Z. Fang, K. Yoon, B. Dizman, R. Weimer, B. P. Rae, X. Li, C. Rader, D. Standaert and W. Olanow, Eur. Polym. J., 2017, 88, 524-552.

25. Y. Li, M. Vergaelen, E. Schoolaert, R. Hoogenboom and K. De Clerck, Eur. Polym. J., 2019, 112, 24-30.

26. Y. Li, M. Vergaelen, X. Pan, F. E. Du Prez, R. Hoogenboom and K. De Clerck, Macromolecules, 2018, 51, 6149-6156.

27. P. Šrámková, A. Zahoranová, Z. Kroneková, A. Šišková and J. Kronek, J Polym Res, 2017, 24, 82.

28. Y. Chujo, K. Sada and T. Saegusa, Macromolecules, 1990, 23, 2693-2697.

29. Y. Imai, K. Naka and Y. Chujo, Polymer Journal, 1998, 30, 990995.

30. M.-J. Shieh, C.-L. Peng, W.-L. Chiang, C.-H. Wang, C.-Y. Hsu, S.-J. J. Wang and P.-S. Lai, Mol. Pharm., 2010, 7, 1244-1253.

31. L. Korchia, C. Bouilhac, V. Lapinte, C. Travelet, R. Borsali and J.-J. Robin, Polymer Chemistry, 2015, 6, 6029-6039.

32. S. Emami and S. Dadashpour, Eur. J. Med. Chem., 2015, 102, 611-630.

33. L. Aoife, Current Pharmaceutical Design, 2004, 10, $3797-$ 3811.

34. U. Till, L. Gibot, C. Mingotaud, P. Vicendo, M. P. Rols, M. Gaucher, F. Violleau and A. F. Mingotaud, Nanotechnology, 2016, 27, 315102.

35. L. Korchia, V. Lapinte, C. Travelet, R. Borsali, J.-J. Robin and C. Bouilhac, Soft Matter, 2017, 13, 4507-4519.

36. D. Rayeroux, C. Travelet, V. Lapinte, R. Borsali, J.-J. Robin and C. Bouilhac, Polymer Chemistry, 2017, 8, 4246-4263.

37. B. Boutevin and Y. Pietrasanta, in Comprehensive Polymer Science, Pergamon Press, New York, 1989, vol. 3, pp. 185194.

38. K. Wang, Y. Liu, W.-J. Yi, C. Li, Y.-Y. Li, R.-X. Zhuo and X.-Z. Zhang, Soft Matter, 2013, 9, 692-699. 
39. Y.-C. Wang, Y. Li, T.-M. Sun, M.-H. Xiong, J. Wu, Y.-Y. Yang and J. Wang, Macromol. Rapid Comm., 2010, 31, 1201-1206.

40. Y. Han, J. Li, M. Zan, S. Luo, Z. Ge and S. Liu, Polymer Chemistry, 2014, 5, 3707-3718.

41. J. Ehrhart, A.-F. Mingotaud and F. Violleau, J. Chromatogr. A, 2011, 1218, 4249-4256.

42. U. Till, M. Gaucher-Delmas, P. Saint-Aguet, G. Hamon, J.-D. Marty, C. Chassenieux, B. Payré, D. Goudounèche, A. F. Mingotaud and F. Violleau, Anal. Bioanal. Chem., 2014, 406, 7841-7853.

43. P. Xin-Mei, L. V. D. Guri and Z. Cheng- He, Current Pharmaceutical Design, 2013, 19, 3884-3930.

44. S. Devulapally, G. Chandraiah and D. Pramod Kumar, MiniReviews in Medicinal Chemistry, 2018, 18, 113-141.

45. L. Korchia, C. Bouilhac, A. Aubert, J. J. Robin and V. Lapinte, RSC Advances, 2017, 7, 42690-42698.

46. X. Zhang, Y. Wang, G. Li, Z. Liu, Z. Liu and J. Jiang, Macromol. Rapid Comm., 2017, 38, 1600543.

47. H. He, Y. Ren, Y. Dou, T. Ding, X. Fang, Y. Xu, H. Xu, W. Zhang and Z. Xie, RSC Advances, 2015, 5, 105880-105888.

48. R. Yang, F. Meng, S. Ma, F. Huang, H. Liu and Z. Zhong, Biomacromolecules, 2011, 12, 3047-3055.

49. Y. Shi, R. van der Meel, B. Theek, E. Oude Blenke, E. H. E. Pieters, M. H. A. M. Fens, J. Ehling, R. M. Schiffelers, G. Storm, C. F. van Nostrum, T. Lammers and W. E. Hennink, ACS Nano, 2015, 9, 3740-3752.

50. M. Taleli, M. Iman, A. K. Varkouhi, C. J. F. Rijcken, R. M. Schiffelers, T. Etrych, K. Ulbrich, C. F. van Nostrum, T. Lammers, G. Storm and W. E. Henninck, Biomaterials, 2010, 31, 7797-7804.

51. A. W. Du, H. Lu and M. H. Stenzel, Biomacromolecules, 2015, 16, 1470-1479.

52. H. Lu, R. H. Utama, U. Kitiyotsawat, K. Babiuch, Y. Jiang and M. H. Stenzel, Biomaterials Science, 2015, 3, 1085-1095. 
Coumarin-Polyoxazoline

\section{Photodynamic therap $\bar{y}$}

Coumarin crosslinked polyxazoline-based vectors developed for efficient photodvinar 